\title{
Structured Regularized Robust Coding for Face Recognition
}

\author{
Xing Wang, Meng Yang*, Linlin Shen \\ College of Computer Science and Software Engineering, Shenzhen University, Shenzhen, China \\ *Corresponding Author; E-mail: yang.meng@ @zu.edu.cn
}

\begin{abstract}
The sparse representation based classifier (SRC) has been successfully applied to robust face recognition (FR) with various variations. To achieve much stronger robustness to facial occlusion, regularized robust coding (RRC) was proposed by designing a new robust representation residual term. Although RRC has achieved the leading performance, it ignores the structured information (i.e., spatial consistence) embedded in the occluded pixels. In this paper, we proposed a novel structured regularized robust coding (SRRC) framework, in which a weight value is assigned to each pixel to measure its importance in the coding procedure and the spatial consistence of occluded pixels is exploited by the pixel weight learning (PWL) model. Efficient algorithms were also proposed to fast learn each pixel's weight value. The experiments on face recognition in several representative datasets clearly show the advantage of the proposed SRRC in accuracy and efficiency.
\end{abstract}

Keywords: Structured regularized; Robust coding; Sparse representation; Pixel weight learning; Face recognition

\section{Introduction}

Face recognition (FR) is one of the hottest research topics in computer vision and pattern recognition due to its wide range of applications such as law enforcement, information security and surveillance etc. In the past two decades FR has been extensively studied [5][23][24], and many representative methods, such as Eigenfaces [6], Fisherfaces [6], LBP [7], have been proposed. Besides, to meet the requirement of practical FR systems, active appearance model [25][26] and active shape model [27] were introduced to handle face alignment. LBP [7] and its extensions [28-31] were used to extract variation-robust texture features. To cope with the illumination variations, Cheng et al. [32] presented a robust face recognition method based on illumination invariant feature in nonsubsampled contourlet transform domain. Wu et al. [33] generalized the Weber-face to multi-scale versions for illumination-robust face recognition. Based on the facial symmetry Hsieh and Tung [34] proposed to utilize the shadow compensation to overcome illumination changes. In terms of dealing with facial occlusion, Eigenimages [8-9], probabilistic local approaches [10], Markov random fields [19] and level set method [35] were proposed for FR with occlusion. A robust kernel 
representation model with statistical local features was developed to handle misalignment, pose variation, and occlusion in face images [36]. In addition, statistical learning of local features [37] was proposed for FR with partial variations, such as expression and occlusion. Although much progress has been made, robust FR to occlusion/disguise still remains challenging due to the complex occlusion variations such as different categories of disguises and the unknown intensity of occluded pixels.

Inspired by studies of the role of parsimony in human vision system [39] [40] and the successful application of sparse presentation in compressive sensing [41] and image restoration [42], Wright et al. [1] originally applied sparse presentation to FR and proposed the sparse representation based classifier (SRC). SRC assumes that samples of different subjects lie on different low dimensional subspaces. In SRC, a testing sample is sparsely coded over all training samples of all subjects and $l_{1}$-norm is imposed on the coding coefficients to guarantee the sparsity. Then the testing sample is classified into the class yielding the minimal reconstruction residual. In addition, SRC uses $l_{1}$-norm to characterize the coding residuals to deal with FR with occlusion. Shortly afterward, Zhang et al. [2] argued that what actually matters is not the $l_{1}$-norm regularization on the coding coefficients but the collaborative representation mechanism (i.e. using all training samples to collaboratively represent the testing sample) and proposed the collaborative representation based classifier (CRC) using $l_{2}$-norm regularization on the coding coefficients. It was shown in [2] that CRC can achieve comparable performance with SRC for FR without outlier pixels but is more efficient. The limitation of CRC is the absence of mechanisms to handle FR with occlusion.

Following SRC and CRC, many representation based works have been proposed for the general robust representation problem or the specific FR with occlusion problem. [18] developed a half-quadratic (HQ) framework to solve the general robust sparse representation problem. The proposed framework unifies algorithms for error correction and detection by defining different kinds of half-quadratic functions, specifically using the additive form for error correction and multiplicative form for error detection, respectively. [17] proposed a structured sparse error coding (SSEC) model to explore the intrinsic structure of the occlusion error in the face image. Specifically, it uses a morphological graph model for the spatial continuity and the shape of the occlusion error and a exponential probabilistic model for the error distribution. Du et al. [43] presented a graph regularized low-rank sparse representation recovery (GLRSRR) method to tackle the case where the facial occlusion may exist in both the training samples and the test sample. Different from SRC assuming the sparsity of the coding residuals, He et al. [11] used a Gaussian kernel-based fidelity term to regularize the coding residuals and proposed a correntropy-based sparse representation (CESR) for robust FR. And Gabor feature was also introduced in the framework of SRC to make the occlusion dictionary compressible and enhance the discrimination [12]. In order to handle more general types of facial occlusion, Yang et al. [4] designed a robust representation term and proposed a regularized robust coding (RRC) model, which has attracted much attention in the field due to its state-of-the-art 
performance. In RRC, each pixel is assigned a weight and outlier pixels will be assigned low weights to suppress their influence on the recognition process. [38] presented a robust nuclear norm regularized regression (RNR) model for face recognition with occlusion. RNR borrows the weight function of RRC to assign each pixel a weight and constrains the representation residual with nuclear norm to implicitly exploit the structural information of the error image. And as CRC, RNR uses the $l_{2}$-norm to regularize the coding coefficients.

Although RRC [4], CESR [11] and Gabor-SRC [12] have achieved leading performance in robust face recognition, all of them measure each pixel's representation residual independently without considering the structured information (i.e., spatial consistence) embedded in the 2D image space. In practical face recognition, most of occluded pixels are not independent but spatially consistent (e.g., block occlusion, facial disguise). Here we should note that the spatial consistence is embedded in occluded pixels but not the occluded pixels' values. Fig. 1 gives an example to show the spatial consistence of image pixels and image pixels' values.

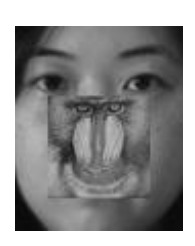

(a)

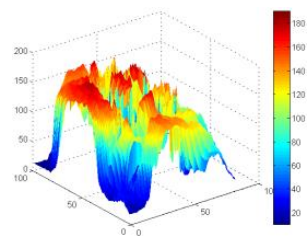

(b)

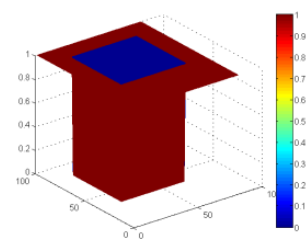

(c)

Fig.1. The structured information of occluded image pixels. (a) a face image with occlusion; (b) pixels' values; (c) pixels' occlusion patterns with $1 \mathrm{~s}$ indicating unoccluded pixels and 0 s indicating occluded pixels. It is easy to see the occluded pixels' patterns but not the occluded pixels' values are spatially consistent.

In this paper, we use a weight to measure a pixel's importance in the coding process, then the structured information could be easily exploited in the pixel weight learning (PWL) model without considering the difference among pixels' values. With the proposed PWL model, the structured information of image pixels could be effectively exploited and a novel framework of structured regularized robust coding (SRRC) was presented for robust FR. We also present efficient algorithms to solve the PWL model. Compared with SSEC [17], HQ [18] and RNR [38], which are also structure regularized based regression methods, the proposed SRRC is very different from them. The difference between SRRC and SSEC is the type of the explored information of the occlusion and the way to explore it. SRRC exploits only the spatial continuity of the occlusion by introducing the PWL model. Whereas both the shape and the spatial continuity information of occlusion are considered in SSEC. One limitation of SSEC is that it does not always converge to the optimal solution. So an additional quality assessment model is used to select the optimal solution from the iteration sequence. The difference between SRRC and HQ lies in the specific research focus. SRRC tries to deal with 
robust FR with occlusion. By contrast, the purpose of HQ is to introduce a more general robust sparse model but ignores the spatial relationship between different image pixels. The difference between SRRC and RNR is the way to exploit the structural information of the occlusion. RNR imposes the nuclear norm constraint on the representation residual to make use of the structural information implicitly, whereas SRRC is more explicit and straightforward. SRRC directly requires the neighboring pixels to have similar weight values through the PWL model to ensure the local consistency of occluded pixels. Furthermore, there is one common difference between SRRC and the other three works, which is the investigation of the type of the norm imposed on the representation coefficients. In this paper we investigate the influence of different regularizations including both $l_{1}$-norm and $l_{2}$-norm on the representation coefficients and conclude that $l_{2}$-norm regularization can achieve comparable performance with $l_{1}$-norm regularization but is much faster. This conclusion is consistent with the previous works, such as CRC [2] and RRC [4]. However, SSEC and HQ only involve $l_{1}$-norm regularization and RNR only involves $l_{2}$-norm regularization. We evaluate the effectiveness of SRRC on several benchmark datasets, such as Extended Yale B [20], CMU Multi-PIE [21], AR [13] and a joint face database of AR [13] and CAS-PEAL [14]. The experiments on these datasets clearly show the advantage of SRRC in both the recognition accuracy and the computational efficiency.

The rest of this paper is organized as follows. Section 2 briefly reviews the related works. Section 3 presents the proposed structured regularized robust coding framework. Section 4 conducts the experiments, and Section 5 concludes the paper.

\section{Related works}

\subsection{Sparse representation based classification (SRC)}

In [1], Wright et al. [1] originally applied sparse presentation to FR and proposed the sparse representation based classifier (SRC). By coding a query sample $\boldsymbol{y}$ as a sparse linear combination of all the training samples via Eq.(1), SRC classifies $\boldsymbol{y}$ by searching for the class that can produce the minimal reconstruction error.

$$
\min _{\alpha}\|\boldsymbol{y}-\boldsymbol{X} \boldsymbol{\alpha}\|_{2}^{2}+\lambda\|\boldsymbol{\alpha}\|_{1}
$$

where $\|.\|_{1}$ is the sparse $l_{1}$-norm and each column vector in $\boldsymbol{X}$ is a training sample. In order to make SRC robust to facial occlusion, an identity matrix $\boldsymbol{I}$ was introduced as an extra dictionary to code the outlier pixels (e.g., occluded pixels).

$$
\min _{\alpha, e}\|\boldsymbol{y}-\boldsymbol{X} \boldsymbol{\alpha}-\boldsymbol{I e}\|_{2}^{2}+\lambda\|\alpha\|_{1}+\lambda\|\boldsymbol{e}\|_{1}
$$


where $\boldsymbol{e}$ is the representation residual vector whose nonzero entries model the occluded pixels. By solving Eq.(2), SRC shows good robustness to FR with occlusion, such as block occlusion and disguise. A theoretical support for the success of SRC is that only a small part of pixels are occluded in most cases. So it is reasonable to require that the representation residual $\boldsymbol{e}$ be sparse.

In SRC, it was emphasized that its powerful performance results from the $l_{1}$-norm sparsity on the coding coefficients. Later on, Zhang et al. [2] reinvestigated the role the $l_{1}$-norm plays in the representation and argued that it is not the $l_{1}$-norm sparsity constraint but the collaborative representation that is really crucial. When only considering the case where there is no outliers, Zhang et al. proposed the collaborative representation based classifier (CRC) which uses $l_{2}$-norm instead of $l_{1}$-norm to regularize the coding coefficients and is expressed as

$$
\min _{\alpha}\|\boldsymbol{y}-\boldsymbol{X} \boldsymbol{\alpha}\|_{2}^{2}+\lambda\|\alpha\|_{2}
$$

The classification of CRC is similar to SRC. Zhang et al. also demonstrated that CRC has competitive performance with SRC but is much faster because CRC has the analytical solution, which is unlike SRC that has to be solved by using expensive optimization techniques. The limitation of CRC is the absence of dealing with FR with outlier pixels.

\subsection{Regularized robust coding model (RRC)}

In order to increase the robustness of SRC to various outliers, He et al. [11] proposed a correntropy-based sparse representation (CESR) for robust face recognition and Yang et al [4] proposed a regularized robust coding (RRC) model. Both CESR and RRC design a robust representation term to discover the occluded pixels. Specifically, RRC improves both the representation residual term $\|\boldsymbol{y}-\boldsymbol{X} \alpha\|_{2}^{2}$ and the sparsity constraint term $\lambda\|\alpha\|_{1}$ in SRC (Eq.(1)) by introducing the following model under the premise that the representation residual of each pixel is independently and identically distributed

$$
\arg \min _{\alpha}\left\{\sum_{i=1}^{n} \rho_{\boldsymbol{\theta}}\left(y_{i}-\boldsymbol{r}_{i} \boldsymbol{\alpha}\right)+\lambda\|\boldsymbol{\alpha}\|_{l_{p}}\right\}
$$

where $n$ is the length of $\boldsymbol{y}$ and is equal to the total number of image pixels, $y_{i}$ is the $i$-th element of $\boldsymbol{y}$ and corresponds to pixel $i$ in the face image, $\boldsymbol{r}_{i}$ is the $i$-th row vector of $\boldsymbol{X}, \rho_{\theta}(e)$ is a robust function of a residual variable $e$, which is defined in [4]. Eq.(4) can be efficiently solved by using an iterative reweighted regularized coding algorithm. By using Taylor expansion of the first term of Eq.(4), in each iteration Eq.(4) changes to

$$
\min _{\boldsymbol{\alpha}}\left\|\operatorname{diag}(\boldsymbol{w})^{1 / 2}(\boldsymbol{y}-\boldsymbol{X} \boldsymbol{\alpha})\right\|_{2}^{2}+\lambda\|\boldsymbol{\alpha}\|_{l_{p}}
$$


$l_{p}$-norm on $\alpha$ could be $l_{1}$-norm or $l_{2}$-norm $\left([2,4]\right.$ indicated that the $l_{2}$-norm regularized coding could achieve the similar accuracy to $l_{1}$-norm but with the faster speed), and $\operatorname{diag}(\boldsymbol{w})$ is a diagonal matrix with the weight vector $\boldsymbol{w}$ as its diagonal vector. Here the element of $\boldsymbol{w}$ is computed as

$$
w_{i}=1 / 1+\exp \left(\mu e_{i}^{2}-\mu \delta\right)
$$

where $e_{i}=y_{i}-\boldsymbol{r}_{i} \boldsymbol{\alpha}$ (please refer to Eq. (4) for $y_{i}$ and $\boldsymbol{r}_{i}$ ), $\mu$ and $\delta$ are two automatically updated scalar parameters in the weight function [4]. Here $w_{i}$ indicates the importance of the $i$-th element of $\boldsymbol{y}$ to the coding of $\boldsymbol{y}$. So $w_{i}$ measures the magnitude of the contribution pixel $i$ in the face image makes to the coding process. We can observe that the outlier pixels will have small weights to reduce their effects on coding $\boldsymbol{y}$ on $\boldsymbol{X}$ since they have big residuals.

When the final coding vector $\boldsymbol{\alpha}$ is achieved, RRC conducts the classification via

$$
\operatorname{identity}(\boldsymbol{y})=\arg \min _{s}\left\|\operatorname{diag}(\boldsymbol{w})^{1 / 2}\left(\boldsymbol{y}-\boldsymbol{X}_{s} \boldsymbol{\alpha}_{s}\right)\right\|_{2}^{2}
$$

where $\boldsymbol{X}=\left[\boldsymbol{X}_{1}, \boldsymbol{X}_{2}, \ldots, \boldsymbol{X}_{c}\right], \boldsymbol{\alpha}=\left[\boldsymbol{\alpha}_{1} ; \boldsymbol{\alpha}_{2} ; \ldots ; \boldsymbol{\alpha}_{c}\right], \boldsymbol{X}_{s}$ is the training samples of class $s, \boldsymbol{\alpha}_{s}$ is the coefficient vector associated with $\boldsymbol{X}_{s}$.

RRC could be solved by alternatively updating the weight vector $w$ and the coding vector $\alpha$ in Eq. (5). The whole algorithm of RRC is briefly summarized in Table 1.

Table 1. Algorithm of RRC.

\section{Solving algorithm of RRC}

1. Initialize $\alpha$

2. Compute residual $\boldsymbol{e}=\boldsymbol{y}-\boldsymbol{X} \boldsymbol{\alpha}$

3. Estimate the weight vector $\boldsymbol{w}$ via Eq.(6)

4. Solve $\alpha$ via the weighted regularized robust coding, i.e., Eq.(5)

5. Go back to step 2 until the update of the weight vector is small enough or the maximal number of iterations is reached

\section{Structured Regularized Robust Coding}

Structured information (e.g., spatial consistence) is embedded in the pixels themselves but not the pixels' values. As shown in Fig.1, the nearby occluded pixels may have quite different intensity values but their occluded patterns are similar, so their weight 
values in RRC are very close. RRC overlooks this structured information because it assumes that each pixel is independent and has independent weight value. By requiring that the weight values in RRC be structured the proposed structured regularized robust coding (SRRC) model could be represented as

$$
\begin{aligned}
& \arg \min _{\alpha}\left\{\sum_{i=1}^{n} \rho_{\boldsymbol{\theta}}\left(y_{i}-\boldsymbol{r}_{i} \boldsymbol{\alpha}\right)+\lambda\|\alpha\|_{l_{p}}\right\} \\
& \text { s.t. } \boldsymbol{w} \text { is spatially structured }
\end{aligned}
$$

where the constraint requires that the weight map of the face image should be spatially structured. Each entry of the map is the weight value of the pixel with the same location as that entry.

Similar to RRC, SRRC can be solved by alternatively updating the coding vector $\alpha$ and the weight value of each pixel. After Taylor expansion of the first term of Eq.(8), in each iteration Eq.(8) changes to

$$
\begin{aligned}
& \min _{\alpha}\left\|\operatorname{diag}(\boldsymbol{w})^{1 / 2}(\boldsymbol{y}-\boldsymbol{X} \boldsymbol{\alpha})\right\|_{2}^{2}+\lambda\|\alpha\|_{l_{p}} \\
& \text { s.t. } \boldsymbol{w} \text { is spatially structured }
\end{aligned}
$$

When the weight vector $\boldsymbol{w}$ is known, Eq.(9) is a weighted regularized coding problem, which can be solved by using existing solvers [4].

When the coding coefficient $\boldsymbol{\alpha}$ is known, firstly the representation residuals are computed as $\boldsymbol{e}=\boldsymbol{y}$-X $\boldsymbol{\alpha}$, and an initial weight vector $\boldsymbol{w}^{0}$ could be calculated via Eq.(6). Then the following pixel weight learning (PWL) model is introduced to figure out the spatially structured weight vector $\boldsymbol{w}$.

$$
\begin{aligned}
& \min _{w}\left\|\boldsymbol{w}-\boldsymbol{w}^{0}\right\|_{l_{q}} \\
& \text { s.t. } \boldsymbol{w} \text { is spatially structured } \\
& \quad w_{i}^{0}=1 / 1+\exp \left(\mu e_{i}^{2}-\mu \delta\right)
\end{aligned}
$$

where the learned weight vector $\boldsymbol{w}$ is required to be not only close to the initial estimation $\boldsymbol{w}^{0}$ but also spatially structured.

We can see that the main difference between SRRC and RRC is that we utilize an additional pixel weight learning (PWL) model (i.e., Eq.(10)) to learn the spatially structured weight vector based on the estimation in each iteration of RRC (i.e., we perform PWL immediately after step 3 of the algorithm of RRC in Table 1). Therefore, the structured information embedded in the occluded pixels can be taken into account.

It is evident that SRRC will degenerate to RRC if there is no structured regularization on $\boldsymbol{w}$. In order to make the robust representation model exploit the structured information, we present the pixel weight learning (PWL) model. 


\subsection{Pixel weight learning (PWL)}

The structured information could be designed in many ways. In this paper, we use the local consistence of image pixels as the structured information. Then the pixel weight learning (PWL) model in Eq.(10) could be rewritten as

$$
\min _{w}\left\|\boldsymbol{w}-\boldsymbol{w}^{0}\right\|_{l_{p}}+\kappa \sum_{i} \sum_{j \in \mathrm{N}_{i}}\left\|w_{i}-w_{j}\right\|_{l_{p}}
$$

where $\kappa$ is a parameter to control the structured regularization, $l_{p}$-norm indicates the $l_{1}$-norm and $l_{2}$-norm when $p=1$ and $p=2$, respectively. For each pixel $i$ in the face image, $j$ is a neighboring pixel of pixel $i$, and $\mathrm{N}_{i}$ is the set of neighboring pixels of pixel $i$.

The second term in Eq.(11) ensures the neighboring pixels have similar weight values. Here the neighboring size could be set by the users. A bigger neighboring region will introduce more global consistence.

Different $l_{p}$-norm regularizations will have different physical meanings. Compared to $l_{2}$-norm, $l_{1}$-norm regularized $\boldsymbol{w}$ - $\boldsymbol{w}^{0}$ could make the learned $w$ not only be similar to $w^{0}$ but also tolerate some sparse difference. When $w_{i}-w_{j}$ is regularized by $l_{2}$-norm, Eq.(11) requires the weight map should be smooth, while $l_{1}$-norm regularization could tolerate some sparse and sharp variance.

Despite that there are four combinations of PWL model with different norms, here we only consider the case that $w_{i}-w_{j}$ is regularized by $l_{2}$-norm since we want the weight values to be spatially consistent in general. Thus the PWL model has two specific forms, PWL $l_{2}$ and PWL $\_l_{1}$. PWL $\_l_{2}$ model could be represented as

$$
\min _{w}\left\|\boldsymbol{w}-\boldsymbol{w}^{0}\right\|_{2}^{2}+\kappa \sum_{i} \sum_{j \in \mathrm{N}_{i}}\left\|w_{i}-w_{j}\right\|_{2}^{2}
$$

And PWL $\_l_{1}$ model could be represented as

$$
\min _{w}\left\|\boldsymbol{w}-\boldsymbol{w}^{0}\right\|_{1}+\kappa \sum_{i} \sum_{j \in \mathrm{N}_{i}}\left\|w_{i}-w_{j}\right\|_{2}^{2}
$$

Compared to the closely related method, i.e., RRC, two advantages can be observed. On one hand, SRRC distributes the weight of each image pixel by considering its neighbors since image pixels are not independent in practice. On the other hand, face image variations (e.g., expression, illumination, occlusion and disguise) are usually spatially continuous, although they have various pixel values. The proposed SRRC can well exploited the spatially structure of face image patterns by using a weight value to indicate a pixel's pattern and a locally continuous constraint on the weight. With the proposed PWL algorithm, it is expected SRRC will have better performance than previous methods. 


\subsection{Solving algorithm of PWL $l_{2}$}

When considering all neighbors of any pixel $i$ in the face image, we can set the size of $\mathrm{N}_{i}$ as $K$ for each pixel (If some neighbor of pixel $i$ is beyond the image boundary we set the weight value of that neighbor as the weight value of pixel $i$ ). In this paper we choose $K=4$ and make $\mathrm{N}_{i}$ include four neighbors above, below, to the left, and to the right of pixel $i$.

In order to efficiently solve Eq.(12), we rewrite the second term of Eq.(12) as

$$
\sum_{i} \sum_{j \in \mathrm{N}_{i}}\left\|w_{i}-w_{j}\right\|_{2}^{2}=\sum_{k=1}^{K}\left\|\boldsymbol{A}_{k} \boldsymbol{w}\right\|_{2}^{2}
$$

where $\boldsymbol{A}_{k} \in \mathbb{R}^{n \times n}$ is a square indication matrix of the $k$-th neighbors of all the image pixels, and $n$ is the total number of image pixels. If the $k$-th neighbor of pixel $i$ is in the image, the $i$-th row vector of $\boldsymbol{A}_{k}$ can be written as

$$
\boldsymbol{A}_{k}(i,:)=\left[\begin{array}{lllllllllll}
0 & \cdots & 0 & 1 & 0 & \cdots & 0 & -1 & 0 & \cdots & 0
\end{array}\right]
$$

Otherwise, the $i$-th row vector of $\boldsymbol{A}_{k}$ is

$$
\boldsymbol{A}_{k}(i,:)=\left[\begin{array}{lllllllllll}
0 & \cdots & 0 & 0 & 0 & \cdots & 0 & 0 & 0 & \cdots & 0
\end{array}\right]
$$

For pixel $i$, in Eq.(15) the entry associated to it is set as 1, while the entry associated to its $k$-th neighbor is set as -1 . It can be seen that $\boldsymbol{A}_{k}(i,:) \boldsymbol{w}$ outputs the weight difference between pixel $i$ and its $k$-th neighbor. So $\boldsymbol{A}_{k} \boldsymbol{w}$ is a vector with each element as the weight difference of a pixel and its $k$-th neighboring pixel.

Let $\boldsymbol{v}=\boldsymbol{w}-\boldsymbol{w}^{0}$, by replacing $\boldsymbol{w}$ with $\boldsymbol{v}+\boldsymbol{w}^{0}$ and according to Eq.(14) we can rewrite Eq. (12) as

$$
\min _{v}\|\boldsymbol{v}\|_{2}^{2}+\kappa \sum_{k}\left\|\boldsymbol{A}_{k} \boldsymbol{v}+\boldsymbol{A}_{k} \boldsymbol{w}^{0}\right\|_{2}^{2}
$$

In this case, we could derive an analytic solution, which could be expressed as

$$
\boldsymbol{v}=-\kappa\left(\kappa \sum_{k} \boldsymbol{A}_{k}^{T} \boldsymbol{A}_{k}+I\right)^{-1} \sum_{k} \boldsymbol{A}_{k}^{T} \boldsymbol{A}_{k} \boldsymbol{w}^{0}
$$

Based on $\boldsymbol{v}=\boldsymbol{w}-\boldsymbol{w}^{0}$, we could further derive

$$
\boldsymbol{w}=\boldsymbol{P} \boldsymbol{w}^{0}
$$

where $\boldsymbol{P}=\left(\kappa \sum_{k} \boldsymbol{A}_{k}^{T} \boldsymbol{A}_{k}+I\right)^{-1}$. Since the indication matrix $\boldsymbol{A}_{k}$ is predefined, the projection matrix $\boldsymbol{P}$ could be pre-computed and in testing stage only a projection operation with a low computation complexity is needed. 


\subsection{Solving algorithm of $P W L \_l_{1}$}

With a similar derivation to PWL $\_l_{2}, \mathrm{PWL} \_l_{1}$ could be rewritten as a sparse coding problem:

$$
\min _{v}\|\boldsymbol{v}\|_{1}+\kappa \sum_{k}\left\|\boldsymbol{A}_{k} \boldsymbol{v}+\boldsymbol{A}_{k} \boldsymbol{w}^{0}\right\|_{2}^{2}
$$

which could be solved by standard sparse coding toolboxes [15]. We denote PWL_l $l_{1}$ which is solved by using a standard sparse coding toolbox by PWL_ $l_{1}$ S. However, these sparse coding solvers would be slow since $\boldsymbol{A}_{k}$ is an indication matrix with a big size.

In order to further improve the efficiency, we develop a faster updating algorithm based on Eq.(13). To solve this problem, we update the weight value one by one. By fixing all the other weight values $w_{i}, i \neq m$, the updating of $w_{m}$ could be rewritten as

$$
\min _{w_{m}}\left|w_{m}-w_{m}^{0}\right|+\kappa \sum_{j \in \mathrm{N}_{m}}|| w_{m}-\left.w_{j}\right|_{2} ^{2}
$$

where $|$.$| means the absolute value. With different cases depending on the sign of w_{m}-w_{m}^{0}$ and by making the derivative of Eq.(21) with respect to $w_{m}$ to be zero, the solution could be derived as

$$
w_{m}=\left\{\begin{array}{cc}
\frac{\sum_{j \in N_{m}} w_{j}}{K}-1 /(2 \kappa K) & \frac{\sum_{j \in N_{m}} w_{j}}{K}-w_{m}^{0}>1 /(2 \kappa K) \\
\frac{\sum_{j \in N_{m}} w_{j}}{K}+1 /(2 \kappa K) & \frac{\sum_{j \in N_{m}} w_{j}}{K}-w_{m}^{0}<-1 /(2 \kappa K) \\
w_{m}^{0} & \text { otherwise }
\end{array}\right.
$$

where as defined in subsection 3.2, $K$ means the number of neighboring pixels of pixel $m$. When the weight values for all the image pixels are updated via Eq.(22), we need several rounds to further update all the weight values to achieve an optimal solution. Since Eq.(22) has a very low computation burden, PWL_ $l_{1}$ could be solved very fast.

\subsection{The whole algorithm of SRRC}

Based on the pixel weight learning (PWL) model, the whole algorithm of SRRC is summarized in Table 2. After the weight vector and the coding coefficients are computed, the classification of SRRC is the same as that of RRC (Eq. (7)). 
Table 2. Algorithm of SRRC.

\section{Solving algorithm of SRRC}

1. Initialize $\alpha$

2. Compute residual $\boldsymbol{e}=\boldsymbol{y}-\boldsymbol{X} \boldsymbol{\alpha}$

3. Estimate the initial weight vector $\boldsymbol{w}^{0}$ via Eq.(6)

4. Learn the spatially structured weight vector $\boldsymbol{w}$ via PWL $l_{2}$ or PWL $l_{1}$

5. Solve $\boldsymbol{\alpha}$ via the weighted regularized robust coding, i.e., Eq.(9)

6. Go back to step 2 until the update of the weight vector $\boldsymbol{w}$ is small enough or the maximal number of iterations is reached

\section{Experimental results}

We conduct experiments on several benchmark datasets, such as Extended Yale B [20], CMU Multi-PIE [21], AR database [13], and a joint database to demonstrate the performance of SRRC. In Section 4.1 we discuss the influence of different neighboring regions used in the PWL model on the FR performance; in Section 4.2, we test SRRC on face recognition with illumination and expression variations; in Section 4.3, we test SRRC on face recognition with random block occlusion; in Section 4.4 we evaluate SRRC on FR with real facial disguise; in Section 4.5 we compare the accuracies and running time on a joint face dataset; in Section 4.6 we illustrate the effectiveness of the PWL model by visualizing the learnt weight maps. The joint database is constructed by using the AR database (100 persons, 2599 images) [13] and a subset of CAS-Peal (101 persons and 843 images) [14]. For the experiments of FR without occlusion, we estimate the weight values of the original face image and then use principal component analysis (PCA) technique to reduce the feature dimensionality, which is like RRC [4].

In all experiments $\kappa$ is empirically set as 0.6 for PWL $l_{1} . \kappa$ of PWL $l_{2}$ is set as 0.05 in face recognition without occlusion and 0.2 in face recognition with occlusion, respectively. As suggested in RRC [4] $\lambda$ in Eq.(9) is set as 0.001. The competing methods include two baseline methods, such as Nearest Neighbor (NN) and Support Vector Machine (SVM), and some latest methods, such as LLC [22], SRC [1], Gabor-SRC [12], CESR [11], HQ [18], RNR [38], RRC_L L [4] and RRC_L 2 [4]. As [18] we denote HQ with the additive form and the multiplicative form as HQSA and HQSM, respectively. We denote SRRC with PWL $l_{2}$ by SRRC2 and SRRC with PWL $l_{1}$ by $S R R C 1$, respectively. Similar to RRC, SRRC1_L $L_{1}, \mathrm{SRRC1} 1 \mathrm{~L}_{2}, \mathrm{SRRC2} \mathrm{L}_{1}$ and $\mathrm{SRRC2} \mathrm{L}_{2}$ represent SRRC1 and SRRC2 using $l_{1}$-norm and $l_{2}$-norm on $\boldsymbol{\alpha}$, respectively. In addition, we also report the results of PWL $l_{1} \mathrm{~S}$ (PWL $l_{1} \mathrm{~S}$ 
means PWL_ $l_{1}$ which is solved by using a standard sparse coding toolbox. Please refer to Section 3.3 for its definition). SRRC1S_L 1 and SRRC1S_L $L_{2}$ are used to represent PWL_ $l_{1} \mathrm{~S}$ using $l_{1}$-norm and $l_{2}$-norm on $\alpha$, respectively.

\subsection{Discussion on the size of neighboring region}

The size neighboring region has an important effect on the generation of weight map and final performance. When we choose a big-size neighboring region, a large-area spatial consistence will be achieved. In that case, a coarse estimation of face pattern (e.g., occlusion) will be generated, but fine face patterns and occlusion boundary information may be lost. When we choose a small-size neighboring region, the fine spatial consistence in a small region can be achieved. Although more iterations may be needed to get a nearly global spatial consistence, the important structure of face variation pattern can be preserved. Compared to a small-size neighboring region, the loss of fine face variation pattern will reduce the final performance of face recognition.

We also conduct some tests of face recognition to evaluate the effect of different-size neighboring regions. In the PWL model, the default setting for the neighborhood is that each pixel's 4 neighbors above, below, to the left, and to the right of the pixel are used. In this section we vary the neighborhood radius from 1 to 5 and use all the neighbors within the specified radius to evaluate the influence of difference neighboring regions. When the radius is 1 there are 8 used neighbors in total. Accordingly, there are 24,48 and 80 neighbors for radius 2, 3 and 4, respectively. The experiments are conducted on the joint database, which has more complex occlusion (Please refer to Section 4.5 for details about the joint database). Table 3 shows FR performance of SRRC using $l_{1}$-norm on $\alpha$ with different neighborhood regions. From Table 3 we can see that when the neighborhood radius is 1,8 nearest neighbors can improve the performance over 4 neighbors in most cases. However, as the neighbor region continues to expand the performance

drops gradually. This may be because a larger neighbor region will introduce more adverse influence in the local region near the occlusion border, where a larger neighbor region will force more pixels outside of the occlusion to have similar weigh values to the pixels inside the occlusion. 
Table 3. Recognition rates (\%) of different neighborhood regions on the joint database with three facial disguises.

(a) Sunglass

\begin{tabular}{lccccc}
\hline Neighborhood radius(Neighbor number) & $1(4)$ & $1(8)$ & $2(24)$ & $3(48)$ & $4(80)$ \\
SRRC2_L 1 & $\mathbf{9 3 . 1}$ & 92.5 & 92.7 & 91.8 & 89.6 \\
SRRC1_L 1 & 94.9 & $\mathbf{9 5 . 5}$ & 93.9 & 89.8 & 89.8 \\
SRRC1S_L 1 & $\mathbf{9 6 . 1}$ & $\mathbf{9 6 . 1}$ & 94.9 & 89.8 & 89.8 \\
\hline
\end{tabular}

(b) Scarf

\begin{tabular}{lccccc}
\hline Neighborhood radius(Neighbor number) & $1(4)$ & $1(8)$ & $2(24)$ & $3(48)$ & $4(80)$ \\
SRRC2_L 1 & $\mathbf{8 3 . 3}$ & $\mathbf{8 3 . 3}$ & 81.6 & 79.8 & 77.4 \\
SRRC1_L 1 & 85.3 & $\mathbf{8 6 . 7}$ & 85.1 & 77.3 & 77.3 \\
SRRC1S_L & 87.6 & $\mathbf{8 8 . 6}$ & 86.7 & 77.3 & 77.3 \\
\hline
\end{tabular}

(c) Hat

\begin{tabular}{lccccc}
\hline Neighborhood radius(Neighbor number) & $1(4)$ & $1(8)$ & $2(24)$ & $3(48)$ & $4(80)$ \\
SRRC2_L 1 & $\mathbf{7 8 . 3}$ & 75.0 & 73.8 & 67.9 & 67.5 \\
SRRC1_L 1 & 82.1 & $\mathbf{8 3 . 8}$ & 81.3 & 66.3 & 66.3 \\
SRRC1S_L & 85.8 & $\mathbf{8 7 . 1}$ & 85.8 & 67.9 & 67.9 \\
\hline
\end{tabular}

\subsection{Face recognition without occlusion}

AR Database): As [4], a subset (with only illumination and expression changes) that contains 50 male and 50 female subjects was chosen from the AR database [13] in this experiment. For each subject, the seven images from Session 1 were used for training, with other seven images from Session 2 for testing. The images were cropped to $60 \times 43$. For all methods except RNR we use the Eigenface with dimensionality 54, 120 and 300 as the face features, respectively. For RNR we use the original cropped images and don't perform dimensionality reduction because the input of RNR takes the form of the two-dimensional image instead of one-dimensional vector. The FR rates by the competing methods are listed in Table 4 (we arrange the recognition rate of RNR in the column of dimensionality 300 artificially). We can see that SRRC1S_L $L_{1}$ achieves the best performance. In addition, SRRC methods could improve the performance of RRC in most cases. Especially, SRRC1S_L $L_{1}$ outperforms RRC_L $L_{1}$ with visible improvement 
(e.g., 1.4\% with 120-d feature). When 300-d feature is used SRRC with $l_{1}$-norm on $\alpha$ and RNR are comparable but the feature dimensionality of SRRC is much lower. Additionally, we can notice that the $l_{1}$-norm on $\alpha$ is always a little better than $l_{2}$-norm and SRRC1 is comparable to SRRC2.

Table 4. Face recognition rates $(\%)$ on the AR database with illumination and expression variations.

\begin{tabular}{|c|c|c|c|}
\hline Dimension & 54 & 120 & 300 \\
\hline $\mathrm{NN}$ & 68.0 & 70.1 & 71.3 \\
\hline SVM & 69.4 & 74.5 & 75.4 \\
\hline SRC [1] & 83.3 & 90.1 & 93.3 \\
\hline LLC [22] & 80.7 & 87.4 & 89.0 \\
\hline GSRC [12] & 87.1 & 93.0 & 95.3 \\
\hline CESR [11] & 73.0 & 80.0 & 83.5 \\
\hline HQSM [18] & 72.7 & 83.3 & 83.3 \\
\hline HQSA [18] & 70.2 & 76.3 & 75.3 \\
\hline RNR [38] & NA & NA & 97.0 \\
\hline RRC_L $L_{2}$ & 84.3 & 94.3 & 95.3 \\
\hline SRRC2_L 2 & 84.4 & 94.0 & 95.9 \\
\hline SRRC1_L 2 & 85.1 & 94.3 & 95.9 \\
\hline SRRC1S_L 2 & 86.3 & 95.0 & 95.6 \\
\hline RRC_L $L_{1}$ & 87.6 & 94.7 & 96.3 \\
\hline SRRC2_L 1 & 88.4 & 95.9 & 97.0 \\
\hline SRRC1_L 1 & 87.3 & 95.6 & 96.6 \\
\hline SRRC1S_L 1 & 88.7 & 96.1 & 97.4 \\
\hline
\end{tabular}

Multi PIE database): The CMU Multi-PIE database [21] contains images of 337 subjects captured in four sessions with simultaneous variations in pose, expression, and illumination. Among these 337 subjects, all the 249 subjects in Session 1 were used for training. To make the FR more challenging, two subsets with both illumination and expression variations in Sessions 1 and 3 , were used for testing. For the training set, as in [4] and [1], we used the 7 frontal images with extreme illuminations $\{0,1,7,13,14$, 16, and 18 \} and neutral expression (refer to Fig. 2(a) for examples). For the testing set, 4 typical frontal images with illuminations 
$\{0,2,7,13\}$ and different expressions (smile in Sessions 1 and 3) were used (refer to Fig. 2(b) for examples with smile in Session 1 and Fig. 2(c) for examples with smile in Session 3). Here the size of cropped images is $50 \times 41$ and we use the Eigenface with dimensionality 300 as the face feature for all methods except RNR, which uses the original cropped image. Table 5 lists the recognition rates in four testing sets by the competing methods.
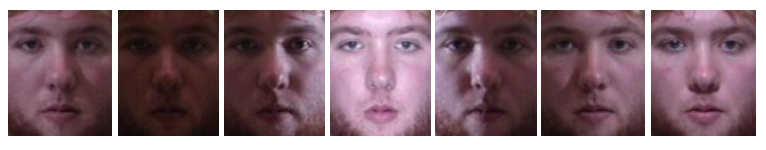

(a)

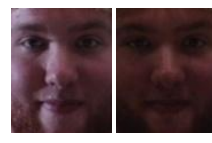

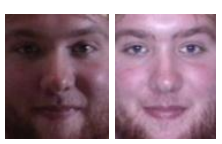

(b)
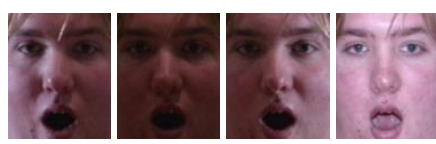

(c)

Fig. 2. A subject in Multi-PIE database. (a) Training samples with only illumination variations. (b) and (c) show the testing samples with smile expression and illumination variations in Session 1 and Session 3, respectively.

Table 5. Face recognition rates (\%) on the Multi-PIE database with illumination and expression variations. ('Smi-S1': set with smile in Session 1; 'Smi-S3': set with smile in Session 3).

\begin{tabular}{|c|c|c|}
\hline & Smi-S1 & Smi-S3 \\
\hline $\mathrm{NN}$ & 88.7 & 47.3 \\
\hline SVM & 88.9 & 46.3 \\
\hline SRC [1] & 93.7 & 60.3 \\
\hline LLC [22] & 95.6 & 62.5 \\
\hline GSRC[12] & 96.4 & 69.4 \\
\hline CESR[11] & 90.2 & 48.8 \\
\hline HQSM [18] & 89.8 & 46.1 \\
\hline HQSA [18] & 88.8 & 47.0 \\
\hline RNR [38] & 96.9 & 65.9 \\
\hline RRC_L 2 & 95.9 & 67.3 \\
\hline SRRC2_L 2 & 96.2 & 67.8 \\
\hline SRRC1_L & 96.6 & 68.4 \\
\hline
\end{tabular}




\begin{tabular}{lcc} 
SRRC1S_L & 96.2 & 67.3 \\
RRC_L 1 & 97.8 & 76.0 \\
SRRC2_L 1 & $\mathbf{9 8 . 1}$ & 76.6 \\
SRRC1_L 1 & $\mathbf{9 8 . 1}$ & $\mathbf{7 6 . 9}$ \\
SRRC1S_L & 97.8 & 76.0 \\
\hline
\end{tabular}

From Table 5, we can see that $S R R C 1 \_L_{1}$ achieves the best performance in all tests, and SRRC2_L performs the second best. Compared to the baseline method, SRC, SRRC1_L $\mathrm{L}_{1}$ has 4.4\% improvement in Smi-S1 and 16.6\% in Smi-S3, respectively. By virtue of Gabor features, GSRC outperforms SRC much and is comparable to SRRC with $l_{2}$-norm on $\alpha$ but pales in comparison with SRRC with $l_{1}$-norm on $\boldsymbol{\alpha}$. Similarly RNR is also comparable to SRRC with $l_{2}$-norm on $\boldsymbol{\alpha}$ but inferior to SRRC with $l_{1}$-norm on $\boldsymbol{\alpha}$. Although the improvement of SRRC over RRC is not big due to that there are no occlusions in face images, the introduction of structured information could still bring some benefits. Different from AR database, in this dataset $l_{1}$-norm on $\alpha$ has much better recognition rates than $l_{2}$-norm, such as the improvement of $1.7 \%$ and $8.7 \%$ on average for Smi-S1 and Smi-S3, respectively. But similar to AR database we can still notice that SRRC1 and SRRC2 have the comparable performance.

\subsection{Face recognition with block occlusion}

In this section we test the robustness of SRRC to block occlusion. We also used the same experimental settings as in [1], i.e., Subsets 1 and 2 of Extended Yale B for training, Subset 3 for testing, and replacing a randomly located square block of a test image with an unrelated image, as illustrated in Fig. 3. The face images were resized to $96 \times 84$.
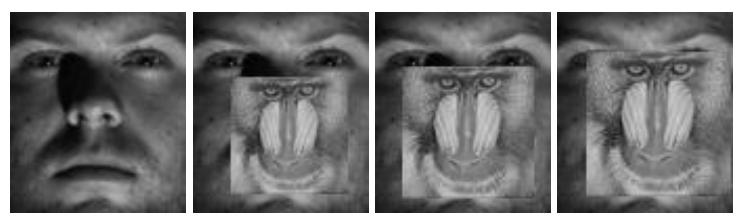

Fig.3. A clean face image and face images with $40 \%, 50 \%$, and $60 \%$ occlusion.

Table 6 lists the detailed recognition rates of the competing methods under the occlusion percentage from $0 \%$ to $70 \%$. Since most of methods could correctly recognize all the test images with $0 \%$ 30\% occlusion, we only report a summarized result. RNR achieves the best performance in all occlusion levels because the rectangular occluded region is low rank. The proposed SRRC1S_L 2 performs the second best, outperform HQSM[18], HQSA[18] and the other competing methods. For occlusion level ranging from $0 \%$ to $40 \%$, the proposed SRRC performs slightly worse than RNR. Compared to RRC, the improvement of SRRC 
becomes bigger as the increase of occlusion percent. For instance, when there is $50 \%$ occlusion, SRRC1_L ${ }_{2}$ outperforms RRC_L 2 by $2.9 \%$, with SRRC1_L $\mathrm{L}_{1}$ outperforming RRC_L $\mathrm{L}_{1}$ by $5.3 \%$ in the case of $60 \%$ occlusion. Compared to GSRC, SRRC still gets better results without using the enhanced Gabor features. We can also observe that SRRC1 is better than SRRC2 in cases with more than $30 \%$ block occlusion, which indicate that there should be some sparse difference between the initial weight and final weight. And it can be noticed that $l_{1}$-norm on $\alpha$ is slightly worse than $l_{2}$-norm in most cases.

Table 6. Face recognition rates (\%) on the Extended Yale B database under different levels of block occlusion.

\begin{tabular}{|c|c|c|c|c|c|}
\hline Occlusion (\%) & $0 \sim 30$ & 40 & 50 & 60 & 70 \\
\hline NN & 86.5 & $\begin{array}{l}62.9 \\
\end{array}$ & 45.7 & 33.1 & 22.3 \\
\hline SRC [1] & 99.6 & 90.3 & 65.3 & 43.5 & 29.2 \\
\hline GSRC[12] & 100 & 96.5 & 87.4 & 64.0 & 32.0 \\
\hline CESR[11] & 90.3 & 75.5 & 57.4 & 37.7 & 19.4 \\
\hline HQSM [18] & 99.8 & 98.5 & 92.5 & 68.4 & 37.5 \\
\hline HQSA [18] & 96.9 & 86.3 & 66.7 & 45.3 & 25.8 \\
\hline RNR [38] & 100 & 99.6 & 95.4 & 84.1 & 49.7 \\
\hline RRC_L $L_{2}$ & 100 & 97.6 & 87.8 & 69.6 & 37.5 \\
\hline SRRC2_L2 & 100 & 98.9 & 89.4 & 70.4 & 40.6 \\
\hline SRRC1_L2 & 100 & 99.1 & 90.7 & 74.6 & 41.9 \\
\hline SRRC1S_L 2 & 100 & 99.3 & 93.2 & 76.4 & 43.7 \\
\hline RRC_L $L_{1}$ & 100 & 96.7 & 87.4 & 69.1 & 38.4 \\
\hline SRRC2_L 1 & 99.9 & 96.5 & 89.4 & 70.4 & 38.9 \\
\hline SRRC1_L 1 & 99.8 & 96.7 & 89.8 & 74.4 & 42.8 \\
\hline SRRC1S_L ${ }_{1}$ & 100 & 96.9 & 91.6 & 75.3 & 41.9 \\
\hline
\end{tabular}

\subsection{Face recognition with real disguise}

A subset from the AR database [13] is used in this experiment. This subset consists of 2,599 images from 100 subjects (26 samples per class except for a corrupted image w-027-14.bmp), 50 males and 50 females. As [1], 799 images (about 8 samples per subject) of non-occluded frontal views with various facial expressions in Sessions 1 and 2 were used for training, while two separate 
subsets (with sunglasses and scarf) of 200 images (1 sample per subject per Session, with neutral expression) were used for testing. All the face images are aligned by using the locations of eyes and cropped to the size of $42 \times 30$. The FR results by the competing methods are listed in Table 7. We can see that RNR and the proposed SRRC are comparable and outperform the other methods. Although CESR and HQ are competitive on FR with sunglass they perform very terribly on FR with scarf. Compared with RRC, SRRC methods achieve higher recognition rates. For instance, SRRC1_L 1 outperforms RRC_L $1, \mathrm{RRC}_{-} \mathrm{L}_{2} 1.0 \%, 2.0 \%$ on FR with scarf, respectively. We can also see that SRRC1 is better than SRRC2, which shows the advantage of PWL_l $l_{1}$ And in this dataset $l_{1}$-norm on $\alpha$ is a little better than $l_{2}$-norm. The proposed SRRC methods also significantly outperform other state-of-the-art methods, including [3] with $84 \%$ on sunglasses and $93 \%$ on scarf, and [16] with $93 \%$ on sunglass and $95.5 \%$ on scarf.

Table 7. Face recognition rates $(\%)$ on the AR database with disguise occlusion.

\begin{tabular}{|c|c|c|}
\hline & Sunglass & Scarf \\
\hline SRC [1] & 87.0 & 59.5 \\
\hline GSRC [12] & 93.0 & 79.0 \\
\hline CESR[11] & 99.0 & 42.0 \\
\hline HQSM [18] & 97.0 & 43.5 \\
\hline HQSA [18] & 97.0 & 39.0 \\
\hline RNR [38] & 99.0 & 100 \\
\hline RRC_L $L_{2}$ & 99.5 & 96.5 \\
\hline SRRC2_L 2 & 99.0 & 97.0 \\
\hline SRRC1_L 2 & 100 & 98.0 \\
\hline SRRC1S_L 2 & 100 & 98.5 \\
\hline RRC_L $L_{1}$ & 100 & 97.5 \\
\hline SRRC2_L 1 & 100 & 97.5 \\
\hline SRRC1_L 1 & 100 & 98.5 \\
\hline SRRC1S_L $L_{1}$ & 100 & 99.0 \\
\hline
\end{tabular}




\subsection{Face recognition on a joint face database}

In this test, we conduct FR with more complex disguises (e.g., sunglasses, scarf and hat) with variations of illumination and longer data acquisition interval. 340 images of the first 85 subjects (4 natural and non-occluded images with different illuminations in Session 1) in AR database and 263 images of the first 80 subjects (the non-occluded images) in CAS-Peal are used as the training sets. And 510 face images with sunglass and lighting variations, 510 face images with scarf and lighting variations, and 240 face images with hat and lighting variations are used as the testing dataset. All the face images are cropped and normalized to the size of 42×30. Some samples are shown in Fig. 4 .
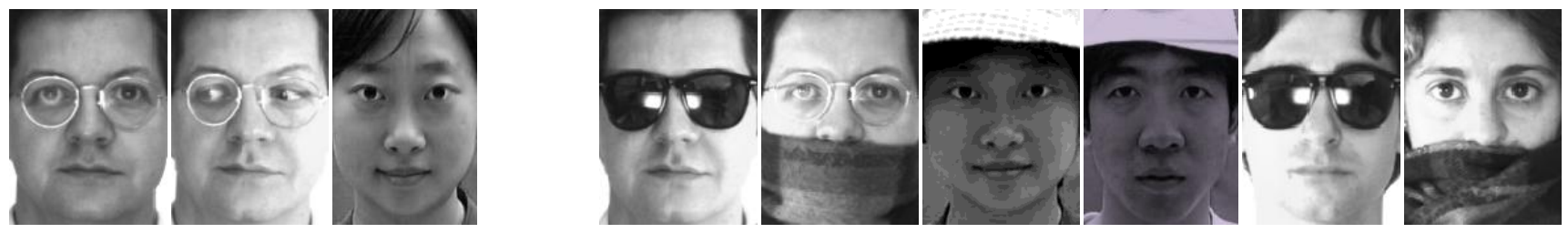

Fig. 4. The training and testing samples in the joint database.

Table 8 lists the results of face recognition on the joint database by competing methods. We can notice that HQ and RNR favour particular types of occlusion. Specifically, HQ is good at the sunglass occlusion but poor at the other types of occlusion, and RNR is s good at the sunglass and scarf occlusion but poor at the hat occlusion. By contrast, the SRRC methods perform well and achieve better results than the other methods in all occlusion types especially in the scarf and hat occlusion (except the case that SRRC2_ $\mathrm{L}_{2}$ is slightly worse than HQ in the sunglass occlusion). Overall, RRC_L $\mathrm{L}_{1}$ achieves the second best performance. SRRC1_L $\mathrm{L}_{1}$ outperforms RRC_L $\mathrm{L}_{1}$ by $4.7 \%, 8.0 \%$, and $15 \%$ in face recognitions with sunglass, scarf and hat, respectively. We can observe that SRRC1 is much better than SRRC2, which shows that for complex disguise occlusion PWL_l $l_{1}$ has the large superiority over PWL $\_l_{2}$. This may be because PWL $\_l_{1}$ allows the large difference between the initial weight and final weight for several pixels so the final weights can be better tuned to adhere to the spatial consistence constraint. And in this experiment $l_{1}$-norm on $\alpha$ is a much better than $l_{2}$-norm.

Table 8. Face recognition rates (\%) on the joint database of AR and CAS-Peal with complex disguise occlusion.

\begin{tabular}{lccc}
\hline & Sunglass & Scarf & Hat \\
\hline SRC [1] & 73.9 & 24.9 & 26.3 \\
GSRC [12] & 52.4 & 66.1 & 34.2 \\
CESR[11] & 80.2 & 11.0 & 26.7
\end{tabular}




\begin{tabular}{|c|c|c|c|}
\hline HQSM [18] & 87.8 & 30.4 & 46.7 \\
\hline HQSA [18] & 88.0 & 23.5 & 45.8 \\
\hline RNR [38] & 84.5 & 81.4 & 41.7 \\
\hline RRC_L 2 & 83.5 & 75.3 & 60.4 \\
\hline SRRC2_L 2 & 87.4 & 81.6 & 71.3 \\
\hline SRRC1_L 2 & 90.8 & 83.5 & 77.5 \\
\hline SRRC1S_L 2 & 91.0 & 85.1 & 83.8 \\
\hline RRC_LL & 90.2 & 77.3 & 67.1 \\
\hline SRRC2_L $L_{1}$ & 93.1 & 83.3 & 78.3 \\
\hline SRRC1_L 1 & 94.9 & 85.3 & 82.1 \\
\hline SRRC1S_L & 96.1 & 87.6 & 85.8 \\
\hline
\end{tabular}

Apart from the recognition rate, computational expense is also an important issue for practical FR systems. In this section, the running time of the baseline method, SRC, and some competing methods which show not bad performance in all cases, including GSRC, RRC_L $L_{2}, R_{R} \_L_{1}$, and SRRC, is evaluated using the FR experiments on the joint face database. In addition, we evaluate the efficiency of RNR because RNR has the incredibly best performance on the Extended Yale B database under different levels of block occlusion (Table 6) and the comparable performance on AR database with disguise occlusion (Table 7). The programming environment is Matlab version R2013a. The desktop used is equipped with a $3.5 \mathrm{GHz}$ CPU and 16G RAM. All the methods are implemented using the codes provided by the authors. For SRC, we use a fast $l_{1}$-minimization solver, ALM [15], to implement the sparse coding step.

Table 9 lists the average computational expense of different methods. We can observe that both $\mathrm{SRRC} 1 \_\mathrm{L}_{2} / \mathrm{SRRC} 2 \mathrm{~L}_{2}$ and RRC_L $L_{2}$ have the least running time, followed by GSRC and SRC. Although the proposed SRRC has similar computation time to RRC, SRRC could achieve much better performance than RRC. Especially, SRRC1_L $L_{2}$ improves RRC_L by 7.3\%, 8.2\% and $17.1 \%$ in Sunglass, Scarf and Hat respectively, and SRRC1_ $\mathrm{L}_{1}$ improves $\mathrm{RRC}_{-} \mathrm{L}_{1}$ by $4.7 \%, 8 \%$ and $15 \%$ in Sunglass, Scarf and Hat respectively. RNR is more time-consuming than SRRC using $l_{2}$-norm on $\alpha$ though it performs better than SRRC under two cases (e.g. on the Extended Yale B database with block occlusion level more than 30\% in Table 6 and on the AR database with scarf occlusion in Table 7). 
Table 9. Average running time (seconds) on the joint database with three facial disguises.

\begin{tabular}{|c|c|c|c|}
\hline & Sunglass & Scarf & Hat \\
\hline SRC (ALM) & 0.610 & 0.579 & 0.574 \\
\hline GSRC & 0.269 & 0.265 & 0.277 \\
\hline RNR & 0.784 & 0.813 & 0.703 \\
\hline RRC_L $L_{2}$ & 0.177 & 0.153 & 0.171 \\
\hline SRRC2_L 2 & 0.200 & 0.170 & 0.194 \\
\hline SRRC1_L & 0.199 & 0.150 & 0.207 \\
\hline SRRC1S_L 2 & 4.05 & 2.81 & 3.43 \\
\hline RRC_L $L_{1}$ & 1.58 & 1.34 & 1.59 \\
\hline SRRC2_L 1 & 1.26 & 1.10 & 1.26 \\
\hline SRRC1_L 1 & 1.40 & 1.17 & 1.41 \\
\hline SRRC1S_L 1 & 5.77 & 3.71 & 4.64 \\
\hline
\end{tabular}

In addition, it is very obvious that SRRC1S_L $\mathrm{L}_{2}$ and SRRC1S_L $\mathrm{L}_{1}$ are extremely time consuming. As mentioned in subsection 3.3 this is caused by using Eq.(20) to solve the PWL_ $l_{1}$ model. Eq.(20) is the standard sparse coding problem with both the large dictionary size and the high dictionary atom dimensionality, which definitely leads to the heavy computational burden. According to Table 8 and all the previous experimental results we can learn that SRRC1_ $\mathrm{L}_{2}$ and $S R R C 1 \_\mathrm{L}_{1}$ can achieve close recognition rates to SRRC1S_L $\mathrm{L}_{2}$ and $\mathrm{SRRC1S} \mathrm{L}_{1}$ in most cases (except for the Hat case in Table 8 where SRRC1S_L $\mathrm{L}_{2}$ is much better than $\mathrm{SRRC1} \_\mathrm{L}_{2}$ ).

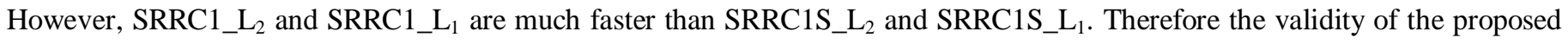
solving algorithm of PWL_ $l_{1}$ is confirmed.

\subsection{Illustrating the effectiveness of the PWL model}

In this section we illustrate the pixel weight learning (PWL) model to demonstrate that it can exploit the spatial consistence of occluded pixels effectively. The experiments are conducted on the joint database. 


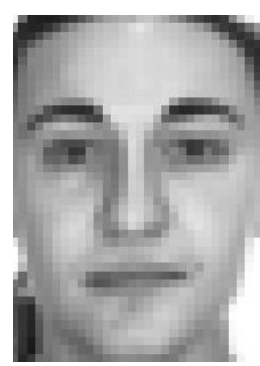

(a)

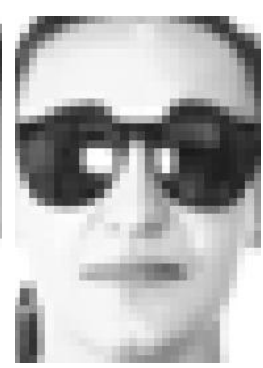

(b)

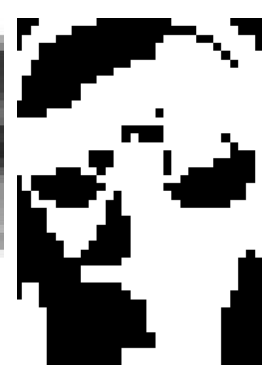

(c)

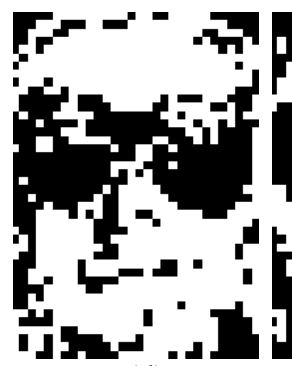

(d)

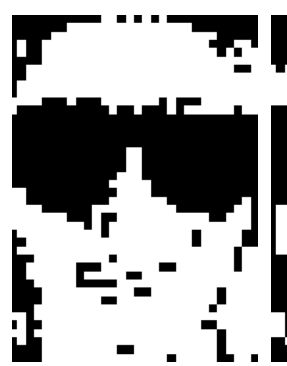

(e)

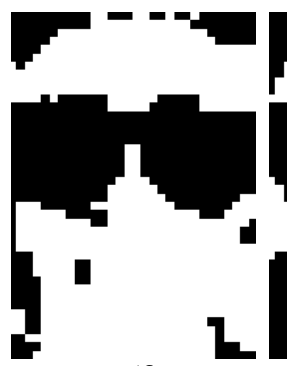

(f)

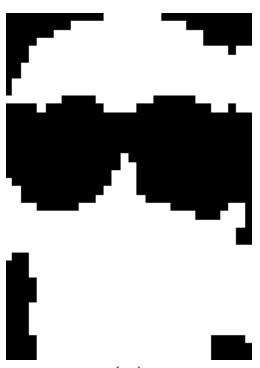

(g)

Fig.5. An example of face recognition with sunglasses disguise. (a) A training image of the identified subject. (b) A testing image with sunglasses. (c) The initial weight map. (d) The weight map when RRC_L ${ }_{1}$ converges. (e) The weight map when SRRC2_L 1 converges. (f) The weight map when SRRC1_L $\mathrm{L}_{1}$ converges. (g) The weight map when SRRC1S_L 1 converges.

Fig. 5 illustrates the proposed PWL model of SRRC by using an example with sunglasses disguise. Fig. 5(a) shows a training image of the identified subject. Fig. 5(b) shows a testing image with sunglasses. Figs. 5(c) shows the initial weight map (binarized for better illustration), which remains the same for $\mathrm{RRC}_{-} \mathrm{L}_{1}, \mathrm{SRRC2} \_\mathrm{L}_{1}, \mathrm{SRRC1} \mathrm{L}_{1}$ and SRRC1S_L . Fig. 5(d), 5(e), 5(f) and 5(g) show the converged weight maps (binarized for better illustration) of RRC_L $\mathrm{L}_{1}, \mathrm{SRRC} 2 \_\mathrm{L}_{1}, \mathrm{SRRC1}_{-} \mathrm{L}_{1}$ and $\mathrm{SRRC1S} \mathrm{L}_{1}$, respectively. We can see that the weigh maps of $S R R C 2 \_L_{1}, S R R C 1 \_L_{1}$ and $S R R C 1 S \_L_{1}$ are more structured and reflect the real-world occlusion truer than that of $\mathrm{RRC}_{-} \mathrm{L}_{1}$, which confirms that the proposed PWL model can exploit the spatial consistence of occluded pixels effectively. In addition, compared with SRRC2_L $\mathrm{L}_{1}$, the weigh maps of SRRC1_L $\mathrm{L}_{1}$ and $\mathrm{SRRC1S} \mathrm{L}_{1}$ are more different from that of $\mathrm{RRC} \_\mathrm{L}_{1}$. This is because SRRC1_L $\mathrm{L}_{1}$ and SRRC1S_L $\mathrm{L}_{1}$ use the PWL_l1 model in which $l_{1}$-norm is used to regularize $\boldsymbol{w}$-w0 in Eq. (13), thus the learned weight vector of SRRC is allowed to tolerate sparse difference from that of RRC.

\section{Conclusion}

This paper presents a novel structured regularized robust coding (SRRC) framework and an associated pixel weight learning (PWL) model for robust face recognition. We also propose effective algorithms to solve the pixel weight learning model. One important advantage of SRRC is that the structured information (e.g., spatial consistence) could be exploited by the proposed SRRC with PWL. Different regularizations in pixel weight learning and the coding coefficients $\alpha$ are analyzed and discussed. Overall, PWL $l_{1}$ is similar to PWL_ $l_{2}$ when there is no obvious occlusion (refer to Table 4 and 5 ) and is better than PWL_ $l_{2}$ when there is large occlusion (refer to Table 6, 7 and 8). And they bear similar and very low computational cost . For SRRC, $l_{1}$-norm on $\alpha$ is a little better than $l_{2}$-norm in most cases, but $l_{2}$-norm is much faster. The proposed SRRC methods were extensively evaluated on FR with various variations, such as illumination, expression, random block occlusion, and real facial disguise. The experimental results clearly demonstrated that SRRC outperforms previous state-of-the-art methods, such as SRC, CESR, GSRC, HQ and RRC. And 
although RNR outperforms SRRC on two cases (e.g. FR with large levels of block occlusion and on the AR database with scarf occlusion) SSRC using $l_{2}$-norm on $\alpha$ is much more efficient than RNR.

\section{Acknowledgement}

This work is partially supported by the National Natural Science Foundation of China under Grants no. 61402289 and 61272050, National Science Foundation of Guangdong Province (Grant no. 2014A030313558), and Shenzhen Scientific Research and Development Funding Program under Grants JCYJ20140509172609171 and JCYJ20130329115750231, Scientific Research Fund for new teachers of Shenzhen University (Grant no. 201536), Scientific Research fund for Advanced Talents of Shenzhen University (Grant no. 000070).

\section{References}

[1] J. Wright, A.Y. Yang, A. Ganesh, S. S. Sastry, and Y. Ma, "Robust face recognition via sparse representation," IEEE Trans. Pattern Analysis and Machine Intelligence, vol. 31, no. 2, pp. 210-227, 2009.

[2] L. Zhang, M. Yang and X.C. Feng, "Sparse representation or collaborative representation: which helps face recognition?" in Proc. ICCV, 2011.

[3] S. Fidler, D. Skocaj, and A. Leonardis, "Combining Reconstructive and Discriminative Subspace Methods for Robust Classification and Regression by Subsampling," IEEE Trans. Pattern Analysis and Machine Intelligence, vol. 28, no. 3, pp. 337-350, 2006.

[4] M. Yang, L. Zhang, J. Yang and D. Zhang, "Regularized robust coding for face recognition,” IEEE Trans. Image Processing, vol. 22, no. 5, pp. 1753-1766, 2013.

[5] W. Zhao, R. Chellppa, P.J. Phillips, and A. Rosenfeld, "Face recognition: A literature survey," ACM Computing Survey, vol. 35, no. 4, pp. 399-458, 2003.

[6] P.N. Belhumeur, J.P. Hespanha, and D.J. Kriengman, "Eigenfaces vs. Fisherfaces: recognition using class specific linear projection,” IEEE Trans. Pattern Analysis and Machine Intelligence, vol. 19, no. 7, pp. 711-720, 1997.

[7] T. Ahonen, A. Hadid, and M. Pietikainen, "Face description with local binary patterns: Application to face recognition," IEEE Trans. Pattern Analysis and Machine Intelligence, vol. 28, no. 12, pp.2037-2041, 2006.

[8] A. Leonardis and H. Bischof, "Robust recognition using eigenimages," Computer Vision and Image Understanding, vol. 78, no. 1, pp. 99-118, 2000. 
[9] S. Chen, T. Shan, and B.C. Lovell, "Robust face recognition in rotated eigenspaces," in Proc. Int'l Conf. Image and Vision Computing New Zealand, 2007.

[10] A.M. Martinez, "Recognizing Imprecisely localized, partially occluded, and expression variant faces from a single sample per class,” IEEE Trans. Pattern Analysis and Machine Intelligence, vol. 24, no. 6, pp. 748-763, 2002.

[11]R. He, W.S. Zheng, and B.G. Hu, "Maximum correntropy criterion for robust face recognition,” IEEE Trans. Pattern Analysis and Machine Intelligence, vol. 33, no. 8, pp. 1561-1576, 2011.

[12] M. Yang and L. Zhang, "Gabor Feature based Sparse Representation for Face Recognition with Gabor Occlusion Dictionary," in Proc. ECCV, 2010.

[13] A. Martinez, and R. benavente, "The AR face database," CVC Tech. Report No. 24, 1998.

[14] W. Gao, B. Cao, S.G. Shan, X.L. Chen, D.L. Zhou, X.H. Zhang, and D.B. Zhao, "The CAS-PEAL Large-Scale Chinese Face Database and Baseline Evaluations," IEEE Trans. on System Man, and Cybernetics (Part A), vol.38, no.1, pp149-161. 2008.1.

[15] A.Y. Yang, A. Ganesh, Z.H. Zhou, S.S. Sastry, and Y. Ma, "A review of fast 11-minimization algorithms for robust face recognition," arXiv:1007.3753v2, 2010.

[16]H. Jia and A. Martinez, "Support vector machines in face recognition with occlusions," In Proc. CVPR, 2009.

[17]X.X. Li, D.Q. Dai, X.F. Zhang, and C.X. Ren, “Structured Sparse Error Coding for Face Recognition With Occlusion,” IEEE Trans. on Image Processing, vol. 22, no. 5, pp. 1889-1999, 2013.

[18]R. He, W.S. Zheng, T. Tan, and Z. Sun, "Half-quadratic based iterative minimization for robust sparse representation,” IEEE Trans. on Pattern Analysis and Machine Intelligence, vol. 36, no. 2, pp. 261-275, 2014.

[19]Z. Zhou, A. Wagner, H. Mobahi, J. Wight, and Y. Ma, "Face recognition with contiguous occlusion using markov random fields," in Proc. ICCV, 2009.

[20] A. Georghiades, P. Belhumeur, and D. Kriegman, "From few to many: Illumination cone models for face recognition under variable lighting and pose,” IEEE Trans. Pattern Analysis and Machine Intelligence, vol. 23, no. 6, pp. 643-660, 2001.

[21] R. Gross, I. Matthews. J. Cohn, T. Kanade, and S. Baker, ”Multi-PIE,” Image and Vision Computing, vol. 28, pp. 807-813, 2010.

[22] J.J. Wang, J.C. Yang, K. Y, F.J Lvx, T. Huangz, and Y.H. Gong, "Locality-constrained Linear Coding for Image Classification", in Proc. CVPR, 2010.

[23] Y.Q. Guo, R. He, W.S. Zheng, X.W. Kong and Z.F. He, "Robust spectral regression for face recognition," Neurocomputing, vol. 118, pp. 33-40, 2013. 
[24] R. He, W.S. Zheng, B.G. Hu, and X.W. Kong, "Two-stage nonnegative sparse representation for large-scale face recognition," IEEE Trans. Neural Network and Learning system, vol. 24, no. 1, pp. 35-46, 2013.

[25] A. Lanitis, C.J. Taylor, and T.F. Cootes, “Automatic Interpretation and Coding of Face Images Using Flexible Models," IEEE Trans. Pattern Analysis and Machine Intelligence, vol. 19, no. 7, pp. 743-756, 1997.

[26] T. Cootes, G. Edwards, and C. Taylor, “Active appearance models,” IEEE Trans. Pattern Analysis and Machine Intellegence, vol. 23, no. 6, pp. 681-685, 2001.

[27] T. Cootes and C. Taylor, “Active shape models - 'smart snakes'," in Proc. BMVC, 1992.

[28] W. Zhang, S. Shan, W. Gao, X. Chen, and H. Zhang, "Local gabor binary pattern histogram sequence (LGBPHS): A novel non-statistical model for face representation and recognition,” in Proc. ICCV, 2005.

[29] B. Zhang, S. Shan, X. Chen, and W. Gao, "Histogram of gabor phase patterns (HGPP): A novel object representation approach for face recognition,” IEEE Trans. Image Process., vol. 16, no. 1, pp. 57-68, 2006.

[30] S. F. Xie, S. G. Shan, X. L. Chen, and J. Chen, "Fusing local patterns of gabor magnitude and phase for face recognition," IEEE Trans. Image Process., vol. 19, no. 5, pp. 1349-1361, 2010.

[31]B. Yang and S.C. Chen, “A comparative study on local binary pattern (LBP) based face recognition: LBP histogram versus LBP image,” Neurocomputing, vol. 120, pp. 365-379, 2013.

[32] Y. Cheng, Y.K. Hou, C.X. Zhao, Z.Y. Li, Y. Hu and C.L. Wang, "Robust face recognition based on illumination invariant in nonsubsampled contourlet transform domain," Neurocomputing, vol. 73, no. 10-12, pp. 2217-2224, 2010.

[33] Y. Wu, Y.Y. Jiang, Y.C. Zhou, W.F. Li, Z.Q. Lu and Q.M. Liao, “Generalized Weber-face for illumination-robust face recognition," Neurocomputing, vol. 136, no. 262-267, 2014.

[34]P.C. Hsieh and P.C. Tung, "Shadow compensation based on facial symmetry and image average for robust face recognition," Neurocomputing, vol. 73, no. 13-15, pp. 2708-2717, 2010.

[35] X. Luan, B. Fang, L.H. Liu, and L.F. Zhou, "Face recognition with contiguous occlusion using linear regression and level set method," Neurocomputing, vol. 122, pp. 386-397, 2013.

[36] M. Yang, L. Zhang, S.C.K. Shiu, and D. Zhang, "Robust kernel representation with statistical local features for face recognition," IEEE Trans. Neural networks and learning system, vol. 24, no. 6, pp. 900-912, 2013.

[37] J. Seo and H.Y. Park, "Robust recognition of face with partial variations using local features and statistical learning," Neurocomputing, vol. 129, pp. 41-48, 2014. 
[38] J. Qian, L. Luo, J. Yang, F. Zhang and Z. Lin, "Robust Nuclear Norm Regularized Regression for Face Recognition with Occlusion," Pattern Recognition, vol. 48, pp. 3145-3159, 2015.

[39] B. A. Olshausen and D. J. Field, "Sparse coding with an overcomplete basis set: a strategy employed by v1? " Vision Research, vol. 37, no. 23, pp. 3311-3325, 1997.

[40] W. E. Vinje and J. L. Gallant, "Sparse coding and decorrelation in primary visual cortex during natural vision," Science, vol. 287, no. 5456, pp. 1273-1276, 2000.

[41]E. Candès, “Compressive sampling,” In Proc. Int. Congress of Mathematics, 2006.

[42] J. Mairal, F. Bach, J. Ponce, G. Sapiro, and A. Zisserman. Non-local sparse models for image restoration. In Proc. ICCV, 2009.

[43]H.S. Du, X.D. Zhang, Q.P Hu, Y.D. Hou, “Sparse representation-based robust face recognition by graph regularized low-rank sparse representation recovery", Neurocomputing, vol. 164, pp. 220-229, 2015. 
Xing Wang currently is a research assistant at the College of Computer Science and Software Engineering, Shenzhen University. He received his B.Eng. and M. Eng. degrees from the College Of Mechanical and Electrical Engineering, Nanjing University of Aeronautics and Astronautics, in 2009 and 2012, respectively. His research interest is sparse representation for face recognition.

Meng Yang is currently an associate professor at School of Computer Science \& Software Engineering, Shenzhen University, Shenzhen, China. He received his Ph.D degree from The Hong Kong Polytechnic University in 2012. Before joining Shenzhen University, he has been working as Postdoctoral fellow in the Computer Vision Lab of ETH Zurich. His research interest includes sparse coding, dictionary learning, object recognition and machine learning. He has published 9 AAAI/CVPR/ICCV/ECCV papers and several IJCV, IEEE TNNLS and TIP journal papers.

Linlin Shen is currently the Director of Computer Vision Institute and professor at School of Computer Science \& Software Engineering, Shenzhen University. He received his Ph.D. degree from University of Nottingham,UK in 2005. Before joining Shenzhen University, he has been working as Research Fellow on MRI brain image processing at Medical school, University of Nottingham. His research interest covers pattern recognition, medical image processing and biometrics. His is the recipient of Most Cited Paper Award by the journal of Image and Vision Computing, the winner of Competition on Cells Classification by Fluorescent Image Analysis organized by ICIP 2013, and Highly Cited Author in China listed by Elsevier in 2015 . 


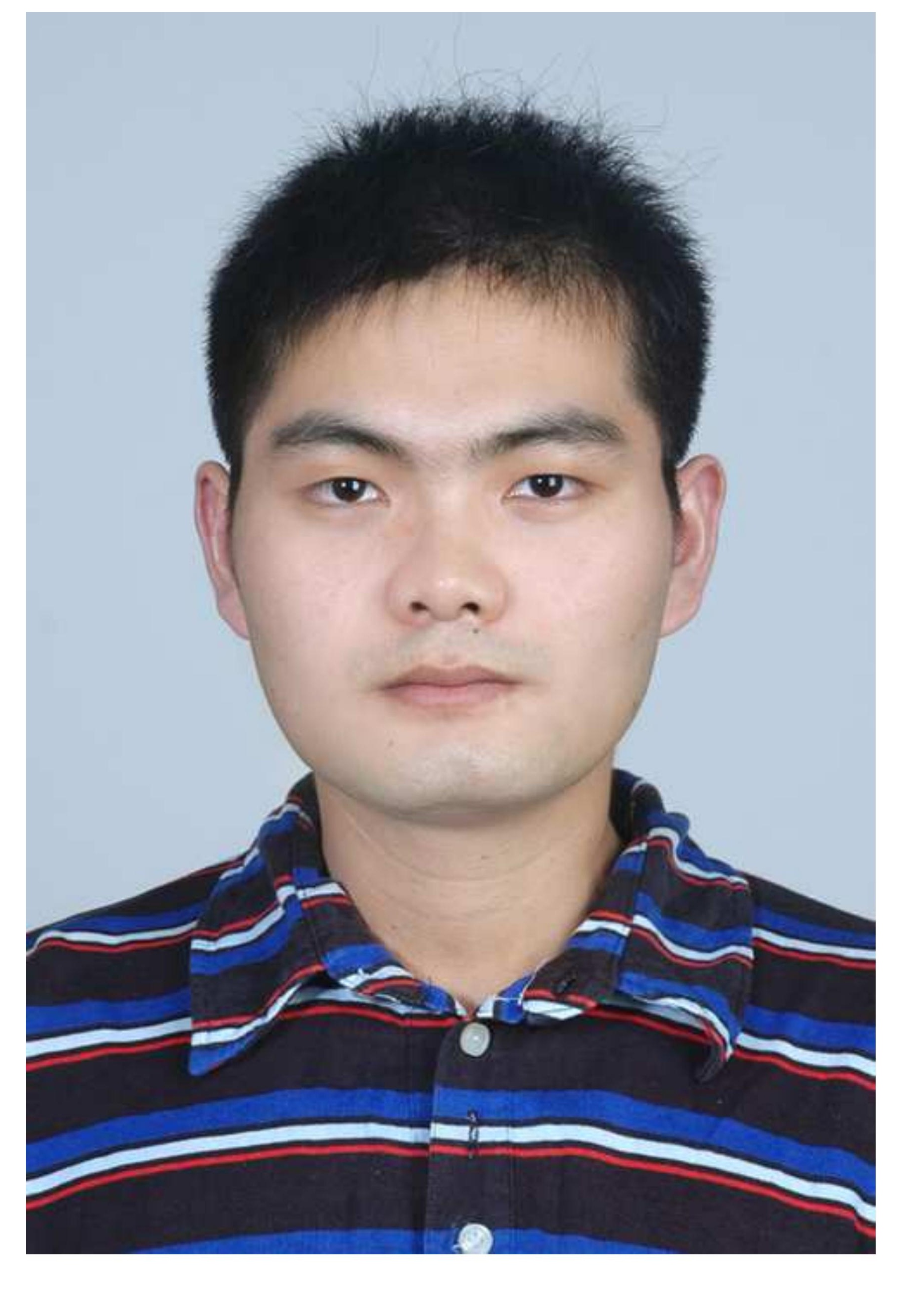

${ }^{*}$ Photo of the author(s)

${ }^{*}$ Photo of the author(s)
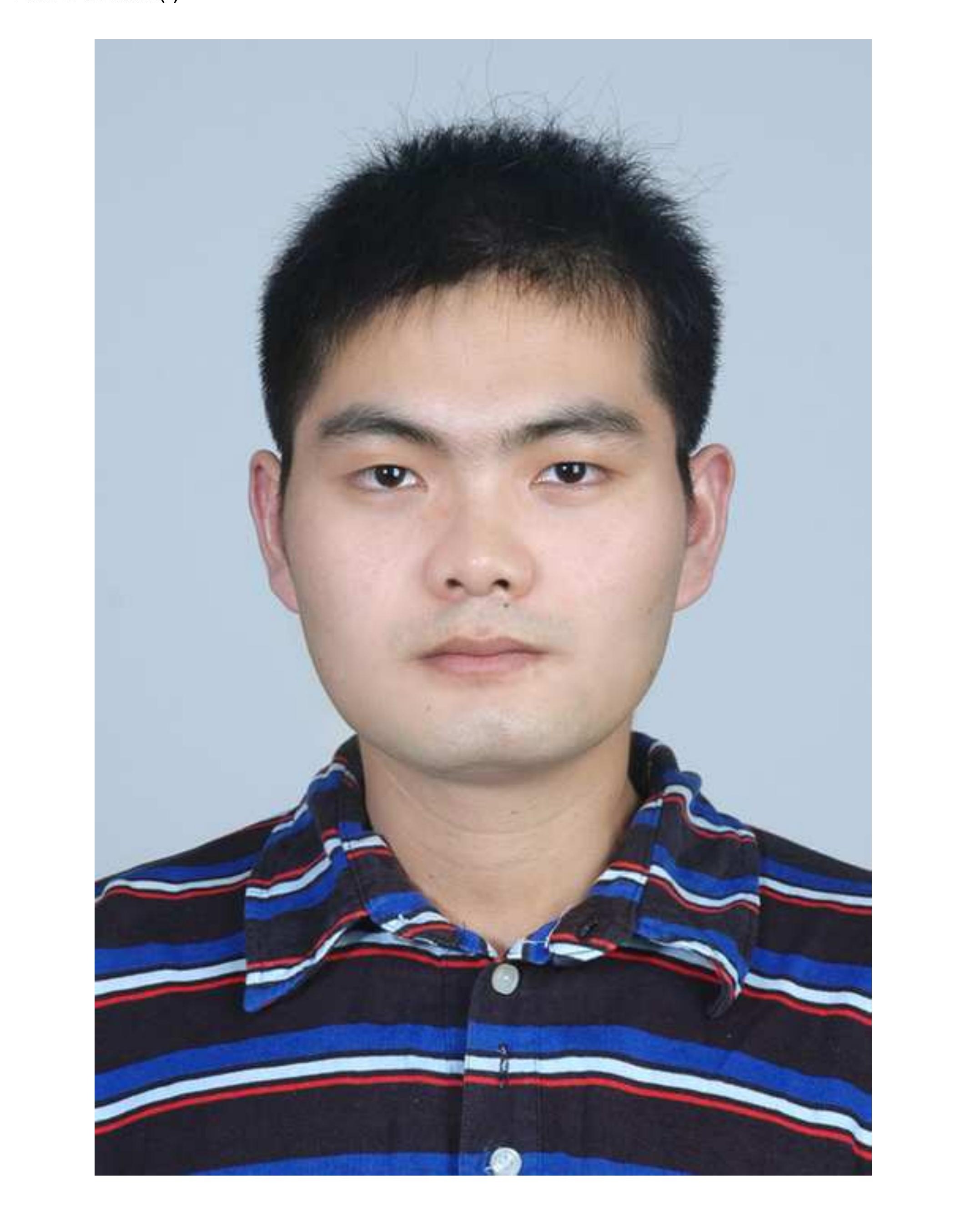


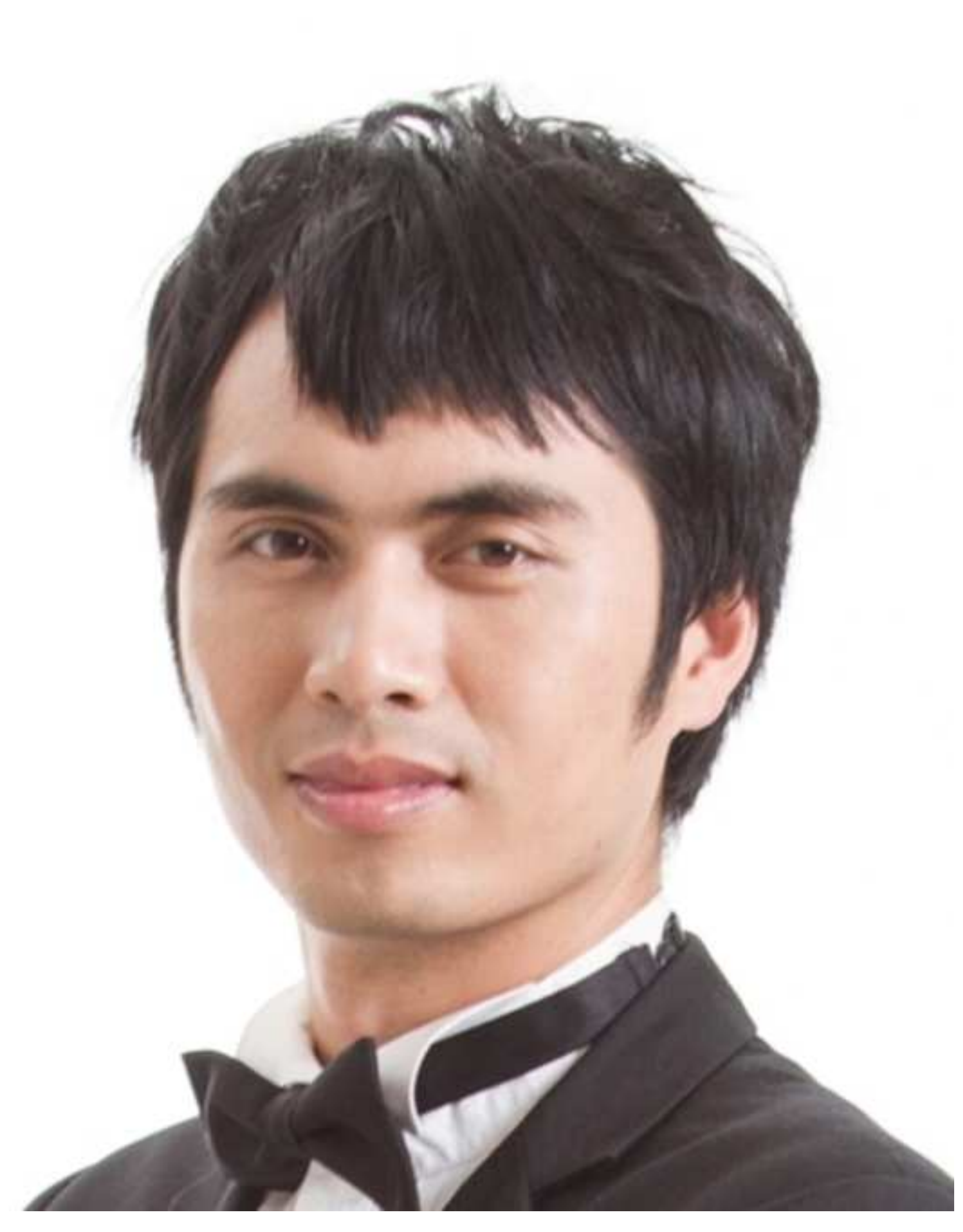

${ }^{*}$ Photo of the author(s)

\section{Photo of the author(s)} )
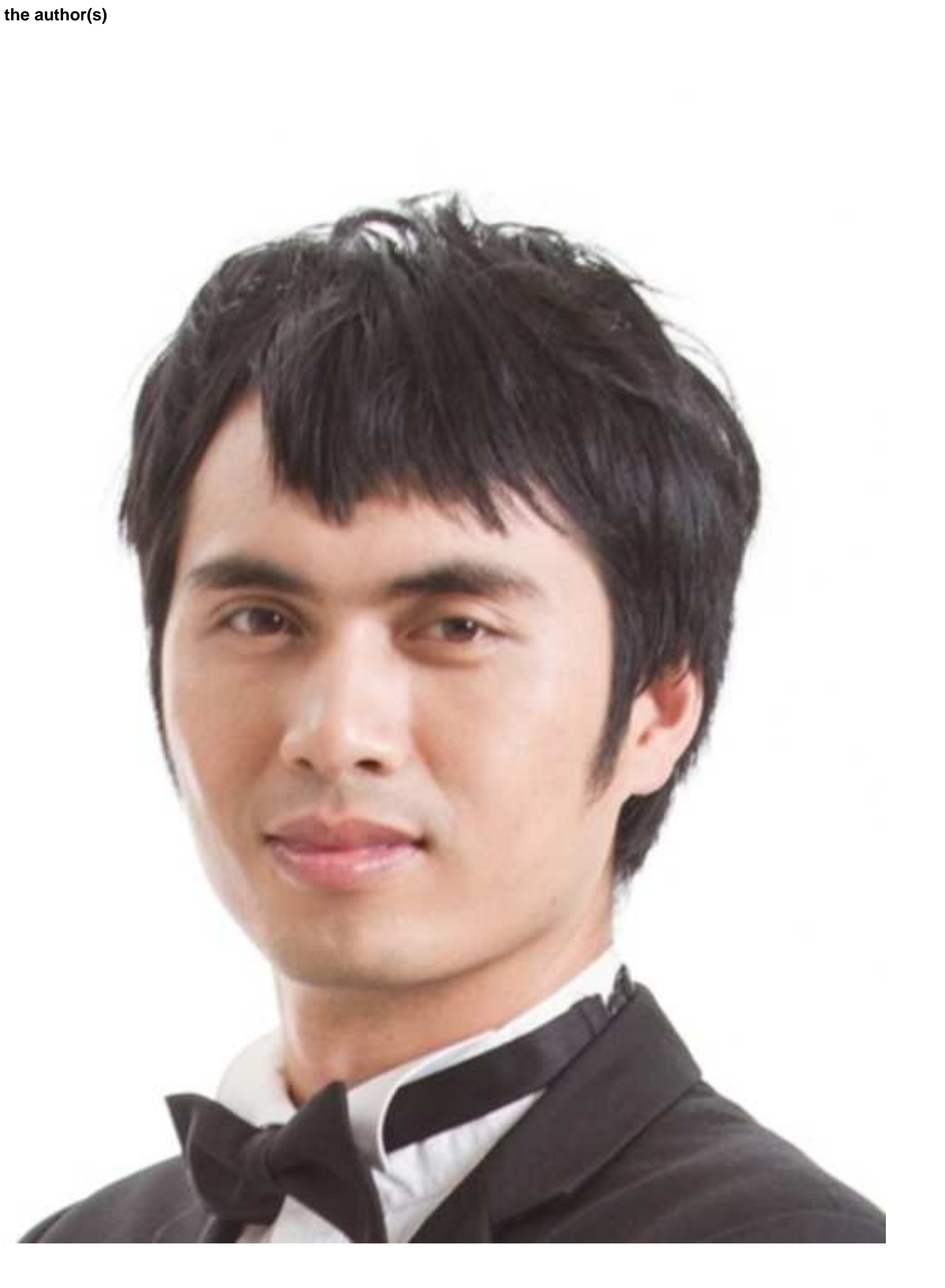


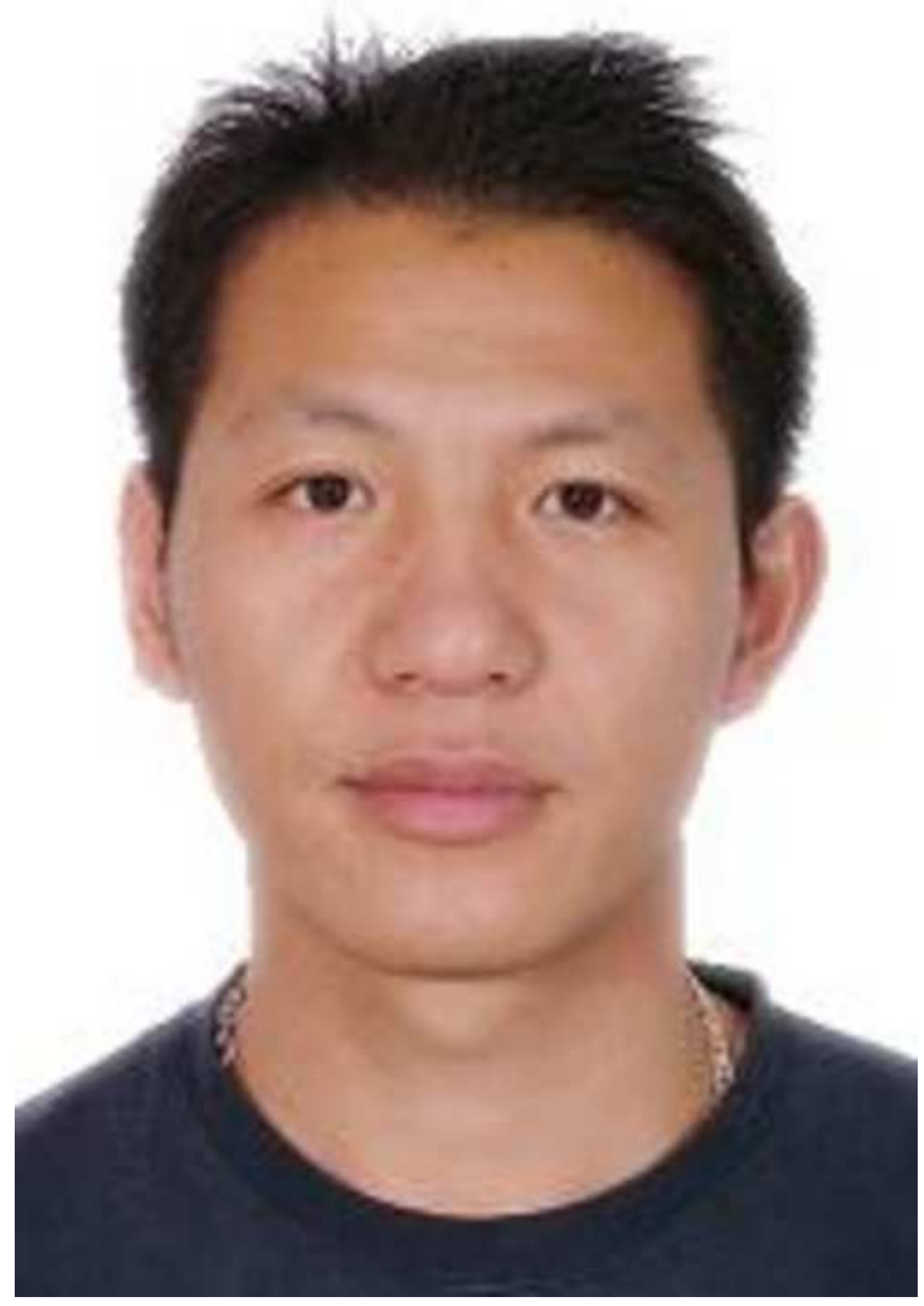

"Photo of the author(s) 